\title{
Cultura Cultura
}

\section{Sensation, affection et corps de chair}

perspectives à partir de Husserl et de Freud

\section{Carlos Morujão}

\section{(2) OpenEdition \\ Journals}

\section{Édition électronique}

URL : http://journals.openedition.org/cultura/2613

DOI : $10.4000 /$ cultura. 2613

ISSN : 2183-2021

\section{Éditeur}

Centro de História da Cultura

\section{Édition imprimée}

Date de publication : 1 décembre 2016

Pagination : 231-245

ISSN : 0870-4546

\section{Référence électronique}

Carlos Morujão, «Sensation, affection et corps de chair », Cultura [Online], vol. 35 | 2016, posto online no dia 09 fevereiro 2018, consultado a 20 abril 2019. URL : http://journals.openedition.org/

cultura/2613; DOI : 10.4000/cultura.2613

Ce document a été généré automatiquement le 20 avril 2019.

(c) CHAM - Centro de Humanidades / Centre for the Humanities 
perspectives à partir de Husserl et de Freud

\title{
Sensation, affection et corps de chair
}

perspectives à partir de Husserl et de Freud

\author{
Carlos Morujão
}

\section{Le but et la méthode}

1 Le but de ma conférence est de présenter les résultats de l'analyse intentionnelle de la manière dont, selon Husserl, se constituent la chose physique, le corps de chair et l'âme. Je suivrai de près les textes de Husserl, mais je me hasarderai parfois à tirer quelques conclusions qui ne sont pas dans les textes et surtout à faire des rapprochements avec Freud. Néanmoins je pense que je serai toujours fidèle à l'esprit de Husserl. À la fin je risquerai une opinion sur l'importance de la théorie husserlienne de l'affection pour comprendre, d'une part la conception que Husserl se fait de l'inconscient, d'autre part, les rapports possibles entre cet inconscient phénoménologique et celui dont nous parle la Psychanalyse.

2 Comme l'indique le titre de ma communication, j'ai l'intention de parler de problèmes qui se rattachent d'abord à la thématique de la sensation - ce sera par ce mot que je traduirai le terme allemand Empfindung - et de l'affect, dans leur rapport avec le corps de chair (qui sera ma traduction de Leib). J'ai été toutefois obligé de faire des choix et de laisser de côté certains problèmes majeurs. À propos de l'Empfindung il y a tout un ensemble de questions qui seraient en elles-mêmes le thème d'une recherche autonome et que je ne pourrai pas approfondir ici. Je pense, par exemple, à l'analyse des rapports entre, d'un côté la doctrine husserlienne de l'Empfindung, qu'on pourra lire dans les Idées II et aussi dans les nombreux écrits sur l'intersubjectivité, particulièrement au volume XIII des Husserliana, et d'un autre côté la doctrine de la hylé dans les Idées I. Il est à mon avis, très intéressant de noter que dans la théorie husserlienne de la constitution du corps de chair et de l'animalité on ne puisse presque pas trouver de références explicites à ce qui avait été dit en Idées I; et que, par conséquent, les très riches suggestions de Husserl pour la 
constitution d'une Anthropologie Philosophique, aussi bien que l'impact de sa théorie de l'Empfindung sur la Psychologie, soient restés presque sans effet sur sa théorie de la constitution des objets matériels qui avait été présentée dans l'oeuvre de $1913 .{ }^{1}$

3 Par rapport à la méthode employée, les analyses suivantes - pas seulement celles de Husserl, mais les miennes aussi, pour autant que je reste fidèle à la méthode husserlienne - supposent la réalisation de la réduction phénoménologique. Je tiens à le dire à l'avance. Pour parler légitimement de la chose physique, de la chair ou de l'âme il faut qu'elles soient données dans une expérience vécue. On ne pourra parler d'elles, sans retomber dans le point de vue de la physique, de la physiologie ou de la psychologie, si on ne prend pas son point de départ dans des actes de consciences dans lesquelles ces objectivités se donnent en tant que telles. La chose physique, la chair et l'âme, malgré toutes leurs différences, sont des unités de sens qui apparaissent à travers une multiplicité d'actes et existent avec la signification qu'elles présentent dans leurs actes. En bref, dans le jargon phénoménologique, je dirai que j'ai l'intention de parler d'elles seulement en tant qu'elles comme noèmes.

Le contenu des analyses qui vont suivre ne s'appuie donc pas ni sur la causalité physique ou physiologique - sur la réceptivité du corps de chair ou sur le seuil d'excitabilité ( Reizbarkeit) des fibres nerveuses - ni sur la causalité psychologique ; quand je parlerai, par exemple, de sensations il faudra toujours comprendre par là des phénomènes de sensations, et quand je parlerai de mouvements cinesthésiques il faudra comprendre qu'il s'agira toujours aussi de phénomènes de mouvement. Les sensations et les cinesthésies nous aurons à les considérer, en tant que phénoménologues, dans leur mode de présentation originaire, et non telles qu'elles sont interprétées scientifiquement par la physiologie ou la psychologie. On pourrait m'objecter que sans cette dépendance d'ordre physiologique ou psychologique nos vécus ne seraient pas ce qu'ils sont. C'est vrai. Ma réponse, cependant, est que je ne pourrai parler de cette même dépendance si elle aussi n'est pas un vécu, quoique d'un autre ordre. Si je fais de la phénoménologie, je n'élimine pas cette dépendance causale, mais j'essaye de comprendre son sens. C'est ce qui justifie aussi l'orientation strictement descriptive que j'ai voulu donner, dans le prolongement de Husserl, aux analyses qui vont suivre.

\section{Lés résultats : la constitution de la chose physique et du corps de chair}

5 Husserl montre - et cette analyse est à mon avis convaincante - que la constitution de la chose physique, de la chair et de l'âme se font à des niveaux différents de la vie intentionnelle. Et tandis que la chair, tout en se fondant sur la chose, a besoin, pour se distinguer d'elle, d'un rapport à une âme, la chose ne requiert pas une autre sphère de fondation. Si toutefois le corps de chair a des propriétés semblables à la chose physique, en tant que, par exemple, il remplit un espace, ces ressemblances ne concernent que leur forme ontologique commune, puisque les niveaux de réalité respectifs sont différents. ${ }^{2}$ C'est en ce sens que au $\$ 20$ de la Leçon de 1907 Chose et Espace ${ }^{3}$ Husserl dit que les distinctions entre materia prima et materia secunda, ou entre l'espace rempli et ses déterminations accessoires (à ne pas confondre avec les soi-disant qualités premières et qualités secondes), valides pour les corps physiques, sont également valides pour le corps de chair. Cependant, en tant que support de champs sensoriels (Sinnesfeldern) il n'est plus simplement qu'une partie d'un monde de choses. 
6 Malgré cela, et dans la mesure qu'il est aussi une chose spatiale, mon corps de chair semble être assujetti à des conditions semblables à celles des corps physiques, quando on en fait l'expérience : il se donne aussi par esquisses et je dois multiplier les perspectives que je puis prendre sur lui pour le constituer dans son identité propre. Si on prend en compte la particularité du rapport en perspective que j'ai avec lui on pourra même l'appeler une chose incomplètement constituée, selon une expression que l'on trouve au $\S$ 41 des Idées II. ${ }^{4}$ Quelques années plus tard, dans la Leçon de 1925 sur la Psychologie Phénoménologique, ${ }^{5}$ Husserl va reprendre le thème et dire que dans toute expérience objective de ce qui est animal ou humain - autrement dit, dès que je prends l'animal ou l'homme en tant que choses spatiales - il y a des fissures ou des failles, ${ }^{6}$ il y a des doutes et des erreurs, parce que les esquisses peuvent ne pas s'accorder toujours entre elles.

Cependant, si on regarde de plus près, les choses sont un peu différentes. Quand il s'agit de mon corps, je ne peux pas m'en approcher ou m'en éloigner, je ne peux pas tourner autour de lui pour voir les parties inaccessibles à la perception directe. Je ne ferai même jamais avec lui cette expérience singulière et fondamentale que Husserl appelle une expérience de déception (Enttäuschung). On peut comprendre ce que je viens de dire à l'aide d'un exemple très simple. Quand je regarde ce que je crois être la façade d'une maison je ne peux pas encore être tout à fait sûr que je suis devant une vraie maison et pas, par exemple, devant un simple décor de cinéma ; j'aurai toujours besoin de tourner autour de cette maison pour en faire la preuve. (Ou bien quelqu'un d'autre l'a déjà fait ou pourra le faire pour moi.) Si mon attente n'est pas confirmée, si je ne voie pas une maison mais un décor, je serai déçu, puisque je m'attendais à voir une maison. Je pourrai faire des expériences semblables à celle-ci avec d'autres corps de chairs (ou de prétendus corps), mais pas avec le mien. J'ai un rapport de nature cinesthésique avec mon propre corps que je ne pourrai jamais avoir avec un autre corps de chair, même si je dois supposer que l'autre sujet qui se présente ${ }^{7}$ à moi à travers ce corps aura tout aussi bien avec lui un rapport cinesthésique semblable. Et aussi quand je me meus ou quand je me déplace il arrive cette chose singulière : tandis que mon corps se meut avec moi, que sa place dans l'espace se change par rapport aux autres choses, moi-même je reste toujours le centre à partir duquel toutes les autres choses sont vues et s'organise aussi la légalité particulière des apparitions en esquisse de chacune d'elles.

8 Les choses physiques sont encore, contrairement au corps de chair, des réalités dépourvues d'histoire, comme Husserl va les appeler au $\$ 33$ d'Idées II. ${ }^{8}$ S'il est possible d'imaginer deux circonstances physiques tout à fait identiques agissant sur mon âme quoique ce soit un fait hautement improbable -, par contre je ne pourrai jamais imaginer deux processus psychiques identiques dans les deux cas, car le premier processus déterminera fonctionnellement le deuxième. Observation très importante je crois, pour comprendre certaines affirmations de Freud. En effet, dans le chapitre VIII d'Inhibition, Symptôme et Angoisse, Freud se refuse à admettre une explication purement causale et physiologique de l'état d'angoisse et fait appel, pour expliquer son étiologie, à ce qu'il nomme les facteurs historiques (ein historisches Moment, dit le texte allemand), ${ }^{9}$ qui ne sont au fond que toute l'histoire antérieure de l'individu dès le moment de sa naissance. Un état d'angoisse actuel reproduit toujours les vécus passés d'un sujet, qui déterminent la quantité d'excitation - et par conséquent de déplaisir - et sa décharge selon certaines voies. (Ces vécus passés dont parle Freud c'est ce qu'on pourrait nommer, en langage husserlien, les sédimentations, auxquelles je reviendrai plus loin.) 


\section{Les résultats : les cinesthésies et le tact}

9 Le corps de chair est constitué comme une unité esthésiologique, autrement dit, il n'est pas une chose qui puisse être affecté par une autre chose selon les simples lois de la causalité physique, mais une réalité qui, en même temps qu'elle est affectée, s'affecte aussi elle-même. Ludwig Landgrebe a caractérisé ce type particulier de réalité à l'aide de l'expression ich bewege mich (je me meus). Cela veut dire : je ne suis pas uniquement affecté, mais je réponds à cette affection par une première forme, certes encore très élémentaire, d'activité intentionnelle. Déjà au niveau de la constitution de la chose physique on peut penser, par exemple, aux mouvements oculaires de contraction ou de dilatation pour mieux voir un objet à distance, ou à ces mouvements de la pupille qui parcourent une surface pour en déterminer l'extension et les limites. Affecté, je tends vers quelque chose en réponse à l'affection, laquelle éveille toujours en moi un intérêt pour ce qui m'affecte. On peut caractériser cette structure intentionnelle, selon Landgrebe, par deux traits fondamentaux, l'attraction et l'éloignement. ${ }^{10} \mathrm{En}$ réalisant des mouvements cinesthésiques je puis, soit être attiré par un objet, soit être repoussé par lui. Il s'agit là d'une structure a priori, antérieure à la distinction entre les actes théorétiques, volitifs ou désidératifs. Attiré ou repoussé, je suis actif ou passif. Toutefois, la distinction entre activité et passivité n'est pas absolue et les limites de l'une et de l'autre sont incertaines.

Mais Husserl va privilégier le sens du tact dans l'analyse de la constitution du corps de chair. Il a un caractère doxique et positionnel qu'aucun autre sens ne possède, ni même le sens de la vision. Je pense qu'on arriverait à la même conclusion par la simple analyse des usages plus courants du verbe sentir, mais on pourra aussi se convaincre de ce privilège si on suit une autre voie. Pensons à la façon dont les sensations tactiles se localisent dans la chair. Si ma main touche une surface rugueuse j'aurai l'expérience vécue de la rugosité. La sensation correspondante est immédiatement localisée dans la chair : c'est ma main droite qui touche, ou une partie de celle-ci, peut-être seulement le bout de mês doigts. Par contre, la sensation visuelle correspond à l'apparition visuelle d'une chose qui ne touche pas mes yeux. C'est pourquoi le tact et les cinesthésies qui lui correspondente ne sont pas seulement un moment du processus de constitution de la chose physique qui est sentie, mais aussi du corps de chair en tant que corps qui sent. Freud aussi a été três sensible à l'importance du tact au point d'attribuer aux tabous portant sur le toucher un caractère plus archaïque que les tabous portant sur le regard. (On peut lire à ce propôs la Deuxième Partie de Totem et Tabou.) C'est avant tout par le toucher, dit Freud, que se manifestent en premier lieu les investissements libidinaux (les Besetzungen), sous forme d'amour ou de haine, comme le prouve, selon lui, l'histoire de l'humanité ou l'étiologie de névroses. Je pense que tous ces rapprochements ne sont pas arbitraires et qu'il s'agit plus que de simples coïncidences. Dans la Leçon n. 31 des Nouvelles Leçons d'Introduction à la Psychanalyse Freud compare le psychisme humain - et il ne parle pas seulement des névrosés ou d'autres malades - à un cristal avec des lignes de fractures; si le cristal se brise, nous nous trouverons devant une maladie, mais la brisure se fait toujours selon des lignes que existent aussi dans le sujet normal. ${ }^{11}$ 


\section{Les sensations et les vécus de sensation}

11 Une petite expérience de pensée nous convaincra aussi de l'importance du tact. Si j'imagine avoir une troisième main - soit par exemple la main gauche de quelqu'un qui est assis à ma droite, main qui se trouve à droite de ma propre main droite -, je ne pourrai pas mouvoir cette hypothétique troisième main par un acte de ma volonté. Avec cette «troisième main» je n'aurai non plus, si je la touche avec une de mes deux mains, la même sensation de toucher et simultanément d'être touché, ce qui arrive toujours quand je touche ma main gauche avec ma main droite. C'est comme le dit Husserl la structure «si... alors...» (wenn... so...) qui fait défaut ici. Dans l'expérience normale de mon corps de chair si je touche la main droite avec la main gauche, alors je me sens en même temps touchant et touché. ${ }^{12}$ Les dimensions physique et esthésiologique de la chair se recouvrent complètement dans cette expérience dont le trait le plus saillant est la réversibilité. J'aurai sans doute la sensation (Empfindung) de cette troisième main si je la touche, mais je n'aurai jamais le vécu de sensation (ce que Husserl appelle Empfindniss) qui caractérise le fait d'être touché.

12 Je m'attarderai un peu sur cette distinction entre Empfindung et Empfindniss, parce que je la trouve très importante. Je traduis, comme je l'ai déjà dit, le premier terme simplement par sensation et le second, comme vous l'aurez remarqué, par vécu de sensation. La sensation c'est ce qui surgit en nous quand nous percevons un corps ; le vécu de sensation c'est ce que nous éprouvons en nous-mêmes en faisant cette perception. Quand je lève un corps lourd j'ai la sensation de son poids ; mais je sens aussi en moi quelque chose de lourd. La sensation renvoi à l'objet perçu, tandis que le vécu de sensation renvoi au sujet qui perçoit, ou mieux, à sa chair.

Les rapports de causalité grâce auxquels mon corps de chair est inséré dans l'apperception normale sont tels que ce même corps, indépendamment de toutes les modifications qu'il éprouve, se maintient dans un cadre d'identité typique. Il est toujours le mien, je l'éprouve comme une partie indissociable de l'unité psycho-physique que je suis moimême. Une brûlure dans ma main peut altérer le sens du tact, mais ne changera pas le fait qu'elle est ma main et que - malgré la douleur - je l'éprouve telle comme avant. ${ }^{13}$

Le vécu de sensation n'est pas simplement une propriété ou un prédicat de la chair quoique la chair, je l'ai déjà dit, ait des propriétés comme toutes les autres choses spatiales. Mes mains, par exemple, ont une certaine coloration ; cette coloration, si je la prends comme une propriété matérielle de mes mains, se donnera par esquisses, selon que je les rapproche ou les éloigne de mes yeux, par exemple, en contractant ou en étendant mês bras. Mais comme le dit Husserl au § 37 des Idées II il ne ferait aucun sens de dire que les vécus de sensation se donnent par esquisses. ${ }^{14}$ Une théorie de la sensation qui ignore que la sensation ne peut être éprouvée par le corps de chair que comme un vécu de sensation est condamnée, comme le rappelait aussi Ludwig Landgrebe, en parlant d'une abstraction, de quelque chose que personne n'a jamais vraiment éprouvée. ${ }^{15}$

\section{Les résultats : la fonction transcendantale du corps de chair}

Ce que je vais dire maintenant n'est peut-être pas tout à fait husserlien, du moins selon la lettre, mais je crois que Husserl nous invite parfois à le lire ou du moins à l'interpréter de la façon dont je vais le faire. Il découle, si ce que je viens de dire à l'alinéa précédent est 
vrai, que la chair joue un rôle transcendantal. Si l'appréhension des données hylétiques se fait selon la structure réceptive d'attraction ou d'éloignement dont j'ai parlé plus haut, nous serons déjà en présence d'une première forme de synthèse, si on consent à donner à ce mot un sens plus large que le kantien. J'appellerai synthèse, en accord, je crois, avec Husserl, tout processus qui tend à la constitution de l'unité d'une multiplicité. ${ }^{16}$ Le corps de chair ne fait pas des synthèses du divers au sens de Kant, mais des fusions continuelles d'éléments qu'il lie par association. Husserl va appeler recouvrement (Deckung) le principe qui est à l'oeuvre dans l'association. Pour que deux sons ou deux couleurs, par exemple, puissent être associés - autrement dit, pour qu'ils se recouvrent - il faut qu'ils se détachent par contraste d'un ensemble homogène d'autres sons et d'autres couleurs et qu'ils acquièrent la force affective suffisante pour réveiller notre attention. Le $\S 28$ et l'Appendice XVIII au volume des Husserliana sur les Synthèses Passives ont des pages três profondes sur cette question. ${ }^{17}$

Sans l'association - sans similitude ou homogénéité sensibles -, sans cette espèce de réverbération ${ }^{18}$ qui émane des objets et qui permet qu'ils se recouvrent même à distance, nous n'aurions pas de monde, mais uniquement un amas chaotique de sensations. On pourrait défendre que le mécanisme associatif s'explique tout simplement par des dispositifs d'ordre biologique dont nous avons besoin en tant qu'animaux pour survivre. Je crois que même si cette hypothèse était vraie, elle ne serait pas pertinente dans le contexte de notre discussion présente. Le sens de cette structure pour l'expérience vécue est indépendant de l'histoire de notre espèce avec son adaptation progressive au milieu. Pour la phénoménologie, l'association est un phénomène d'unité à l'intérieur du courant de la conscience ; quand il a lieu dans une conscience actuelle, c'est grâce au processus de sédimentation d'actes de conscience passés.

Il y a des rapprochements très intéressants à faire avec Freud à propos de ces niveaux encore très élémentaires de l'activité synthétique. Dans ses Leçons d'Introduction à la Psychanalyse Freud compare les investissements libidinaux sur les objets (les Objektbesetzungen) aux pseudopodes de certains organismes unicellulaires, qui sont capables de s'étendre dans la direction des objets par lesquels ils sont attirés, ou de s'en retirer rejoignant le corps de la cellule. ${ }^{19}$ Dans le même sens, dans le petit essai «La Négation» Freud dit que les processus psychiques d'ordre supérieur se déroulent selon une technique qui a déjà été essayée à l'extrémité sensorielle de l'appareil psychique dans le phénomène du tact:

«Selon notre hypothèse, la perception en fait n'est en aucune façon un processus passif, mais le Moi envoi périodiquement au système perceptif de petites quantités d'investissement libidinal, au moyen desquelles il saisit les stimuli externes, pour se retirer ensuite après chaque avancée tactile de ce genre. $»^{20}$

D'ailleurs on trouve dans tout cet essai des affirmations très intéressantes et il serait facile de montrer combien elles s'accordent avec ce que dit Husserl. Par exemple, Freud dit que le jugement est le moment de la décision, qu'il met fin à un état de prolongement indéfini de la pensée (Freud l'appelle Denkaufschub) et est à l'origine d'actions motrices. Husserl dit presque la même chose dans les Analyses pour la Synthèse Passive, en des passages qui ont été repris dans Expérience et Jugement ; aussi bien que Freud, Husserl dit que le jugement est le moment de la décision qui mène à son terme un état de tension non résolu, pendant lequel la pensée flotte (schwebt) devant des alternatives entre lesquelles elle ne réussit pas à se décider. Bien sûr, en tant que phénoménologues nous devrions appeler «naturaliste» la façon de s'exprimer de Freud. Je pense néanmoins que Freud va 
parfois plus loin que ce que son langage laisse supposer. Ce qui est en cause c'est l'existence de mécanismes d'orientation et d'organisation de l'expérience, dont la façon d'opérer est déjà à caractère transcendantal. Pour leur nier ce caractère il faudrait démontrer que Freud défend un rapport tel entre le stimulus et la réponse - ou entre l'excitation et la décharge - qu'à un accroissement ou diminution des premiers correspond toujours un accroissement ou une diminution proportionnels des secondes, ce que, je pense, il ne fait jamais.

\section{Les résultats : le corps et l'âme}

19 Jamais je n'arriverai à percevoir l'âme comme je perçois le corps, c'est-à-dire, en multipliant les perspectives que je puis prendre sur elle. L'âme - ou la psyché - n'est pas d'ailleurs la face cachée d'un corps physique. Si on essaye de transposer la notion de substance des choses à l'âme, on verra que l'âme n'est pas une substance, qu'on ne pourra pas parler d'un en-soi de l'âme qui se présenterait par des esquisses, que l'âme n'est pas une unité schématisée, autrement dit, constituée en résultat d'une synthèse qui résulterait, finalement, de ses apparitions successives.

20 Je ne puis éprouver mon propre psychisme que sur la base de sa relation au corps de chair qui le spatialise et le temporalise. On ne comprendra jamais la nature de l'âme si on ne la saisit pas dans son rapport à sa base extra-psychique. Je parlais au début de mon exposé d'un rapport de fondation. Mais il faut ajouter que l'âme n'est pas localisée dans un corps de la même façon que les sensations tactiles ou comme si elle s'étendait sur lui dans un rapport de recouvrement. Il n'y a pas de champs animiques semblables aux champs sensoriels. Husserl parle d'une introjection de l'âme dans le corps dans les Idées II, un terme qu'il avait utilisé déjà à d'autres effets. Il est vrai, comme Husserl le dira dans les Idées III, ${ }^{21}$ que nous sommes en présence de trois niveaux de réalité : le corps physique, le corps de chair et l'âme, mais ce n'est pas l'âme qu'on doit opposer au corps physique, mais l'unité concrète d'âme et de corps, c'est-à-dire, le corps en tant que corps animé ( beseelte Leib, comme dit le texte allemand).

21 Saisir ce rapport de fondation de l'âme est essentiel pour comprendre le phénomène particulier qu'est la mobilité de notre corps. En conditions normales, nous avons la connaissance immédiate de la localisation dans l'espace de chacune de ses parties e de ce qu'il faut faire avec elles à chaque moment. Je sais quels mouvements je dois faire pour lever un objet du sol et, dans certaines conditions, je saurai même par avance, du moins approximativement, la quantité d'effort nécessaire que j'aurai à dépenser. C'est pourquoi il faudra dire que le corps de chair est finalement plus qu'un centre d'activités cinesthésiques, mais qu'il est déjà un pôle subjectif, bien que ce ne soit pas encore la subjectivité qui réalise des actes. Je pense que c'est une thèse fondamentale de Husserl, à laquelle il revient sans cesse, que l'expérience que j'ai de mon âme, ou de Moi en tant que sujet de propriétés personnelles, est indissociable de l'expérience que j'ai de mon corps. Ils sont d'ailleurs tellement enchevêtrés que je ne puis me trouver sans trouver mon corps. Les structures temporelles de ses deux expériences sont exactement les mêmes, comme le montrent les analyses du cours du semestre d'hiver de 1910-1911 intitulé Problèmes Fondamentaux de la Phénoménologie..$^{22}$ C'est ainsi qu'en tant que je deviens autre dans la durée vécue, je suis encore le même que j'étais à l'instant qui vient d'avoir lieu (ce que Husserl nomme le Soeben) et qui est encore donné dans la rétention; de même, le corps que j'ai maintenant, ou qui est maintenant en tant que mon corps, est le même 
corps qui vient d'avoir été ; et si moi je me retrouve à nouveau dans le ressouvenir, de même le corps ressouvenu est mon corps qui est maintenant.

S'il est légitime de parler d'un excès de réalité du corps de chair par rapport à la chose physique, en tant qu'il est l'unité d'un corps et d'une âme ; s'il n'y a aucun rapport d'essence entre un vécu psychique et une chose physique; et même si je puis penser le plaisir ou la douleur sans rapport à l'étendue chosale, de la même façon que je puis penser un effet sans rapport à sa cause, à laquelle il est pourtant empiriquement toujours lié, ${ }^{23}$ cet excès toutefois n'est pas réellement séparable du physique, mais plutôt fondé sur lui. Un texte daté de 1905 le dit à propos de l'expérience du corps de l'autre : si je touche avec ma main, ou n'importe quelle partie de mon corps, un autre corps de chair, je touche une unité psycho-physique, et quoique le psychisme de l'autre ne me soit pas donné de la même façon que son corps, il ne me serait pas donné du tout sans la présence d'un corps. ${ }^{24}$ Mais jamais, d'un point de vue noématique, je n'aurai là l'apparition de quelque chose que je puisse constituer comme un simple objet.

\section{Les conséquences pour une théorie de l'inconscient}

Il faut toujours se méfier des ressemblances entre le vocabulaire technique de deux penseurs. Qu'ils puissent utiliser souvent les mêmes termes ne nous garantit pas qu'ils parlent de la même chose. Husserl parle couramment de Hemmung (inhibition), de Verdrängung (refoulement) ou d'association, par exemple, dans les Analyses pour la Synthèse Passive. Il le fait après que Freud ait donné à tous ces concepts un sens technique précis, mais il ne veut pas dire exactement la même chose que Freud. Il suffit de faire un peu d'attention aux contextes. Husserl dit, par exemple, que je puis associer par similitude un carré rouge et un carré bleu, mais qu'une association par égalité est inhibée (gehemmt) par la différence des deux couleurs. Ou encore que la perception d'un carré rouge refoule (verdrängt) la perception d'un carré bleu, dès que le premier vient se superposer au second. ${ }^{25}$

En d'autres contextes, par contre, il faut être très attentif aux ressemblances de vocabulaire. Dans les Analyses pour la Synthèse Passive Husserl parle du processus de formation de ce qu'il appelle au $\S 33$ des configurations d'unité (Einheitsbildungen) d'où sortiront plus tard les objets, quand le moi deviendra actif. ${ }^{26}$ Ces Einheitsbildungen ne sont pas encore des objets, mais plutôt des multiplicités de parties associées par similitude ou contraste, ou par une affinité dans la façon dont les diverses parties s'ordonnent entre elles. Mais ces multiplicités peuvent ne pas se constituer, le processus associatif peut ne pas arriver à son terme. Husserl parle alors de la présence de hemmende Gegenpotenzen (de puissances en sens contraire, capables d'inhiber ce processus), dont l'origine et la nature devraient être étudiées par une phénoménologie de «l'inconscient».

Le mot «inconscient» est entre guillemets dans le texte allemand. Ils sont, à mon avis, du moins c'est mon hypothèse, des guillemets de citation. Quand Husserl ne met pas le mot entre guillemets il dit «le soi-disant inconscient». Husserl se réfère donc à une autre conception de l'inconscient, qui ne peut être que celle de la Psychanalyse et de Freud, et qu'il juge intenable. ${ }^{27}$ Peut-être Husserl n'a pas tout à fait raison : le modèle de fonctionnement du psychisme qu'il propose a en commun avec celui de Freud le fait d'admettre que la conscience a devant elle son propre inconscient, que celui-ci n'est pas simplement le reste somatique d'une activité psychique passée, mais, finalement, pour 
me servir des mots mêmes de Freud dans la Leçon 31 des Nouvelles Leçons d'Introduction à la Psychanalyse, un pays étranger à l'intérieur (ein inneres Ausland). ${ }^{28}$

Cette thèse, que Husserl ne formule pas explicitement et qu'il ne se propose pas de démontrer jusqu'au bout, on peut tout de même la retrouver dans ses nombreuses pages inédites. Je m'appuierai surtout sur le texte des Analyses pour la Synthèse Passive (plus précisément, dans le remaniement, fait en 1925, du cours de 1921 sur la Logique Transcendantale) et de la Leçon de 1907, Chose et Espace. (Je ferai aussi quelques références à certains ouvrages de Freud.) En me rapportant à quelques passages de ces textes, j'ai l'intention de démontrer l'impossibilité d'identifier la psyché et la conscience, ce qui est surtout évident si on comprend le sens du phénomène que j'appelle, en me servant des termes mêmes de Husserl, l'obscurcissement des affections.

e commencerai par le texte des Synthèses Passives. ${ }^{29}$ Husserl est en train de faire l'analyse du phénomène que je viens de mentionner et qu'il appelle, comme je l'ai dit, l'obscurcissement ou l'affaiblissement des affections (Verdunkelung c'est le terme allemand qu'il emploie). Il serait tentant immédiatement de le rapprocher au refoulement freudien, mais je pense qu'il faudra finalement suivre une autre voie. Qu'est-ce qui se passe quando une affection s'affaiblit ? Husserl dit que c'est un phénomène qui obéit à une typique, autrement dit, à une structure qui se maintient inaltérable indépendamment de l'intensité ou de la qualité des affections. (Un son très fort ou presque inaudible, un rouge foncé ou un jaune pâle s'affaiblissent de la même façon, si on fait l'analyse de ce processos après la réduction phénoménologique, pour en dégager la légalité.) Toute affection souffre une première modification rétentionelle en étant chassé de la "pointe avancée» du présent vivant, mais le déroulement de ce processus arrive à son terme quand l'affection atteint son point zéro (eine Null, dit Husserl au $\$ 37$ des Synthèses Passives ). On demandera : qu'est-ce que ce zéro où, au bout d'un certain temps, se trouveront toutes mes affections?

Il faudra peut-être revenir en arrière pour comprendre ce que Husserl veut dire, parce qu'au $\S 33$ des Analyses pour la Synthèse Passives, Husserl avait déjà parlé d'un point zéro : ein Nullfall der Weckung, le point zéro de l'être éveillé. Si j'ai bien compris ce texte, Husserl ne parle pas d'une absence d'affection, mais d'une circonstance où l'affection n'a pas la force suffisante pour éveiller l'attention, ou parce qu'elle est trop faible, ou peut-être parce que je l'ai refoulée. Mais Husserl ajoute : ce zéro est un zéro au sens des arithméticiens, c'est-à-dire, le contraire d'une quantité numérique (Anzahl), mais encore un nombre dont en certaines circonstances on doit tenir compte pour calculer. C'est pourquoi Husserl répond à la question que j'ai posée il y a un instant : ce Nullpunkt n'est pas un néant, mais un Réservoir. (C'est le mot français qui apparait dans le texte allemand.) C'est le même mot qu'on rencontre parfois dans les textes de Freud - je pense, entre autres, à Le Moi et le Ça--, qui parle de das grosse Reservoir der Libido, mais dans un sens qui n'est pas celui de Husserl. ${ }^{30}$ Dans le Réservoir husserlien nous rencontrerons toutes les objectivités qui ont déjà été présentes dans un présent vivant qui appartient maintenant au passé, mais qui, contrairement au Réservoir de Freud, seront toujours à la disposition de la conscience, grâce à l'association.

Dans un passage du Chapitre I de Malaise dans la Civilisation Freud dit que c'est três difficile de savoir ce qui arrivera à ce qui m'a un jour affecté (à ce que j'ai vu, entendu ou touché), mais qu'il est très peu probable que cela puisse disparaitre complètement. C'est vrai que Freud ne parle pas, dans ce passage, de toutes les dimensions de ce qu'est pour lui l'inconscient, mais seulement du refoulé. Mais Husserl a des métaphores très 
interessantes pour expliquer ce fait. Il dit, par exemple, que les sédimentations des expériences passées se superposent (übereinanderlegen) de telle façon que les plus anciennes sont totalement recouvertes par les plus récentes. Je pense que c'est à partir de là qu'on pourra trouver une possibilité de rapprochement et de dialogue fructueux entre la phénoménologie et la psychanalyse en ce qui concerne le problème de l'inconscient : quelque chose de plus intéressant que de savoir si cet inconscient phénoménologique finalement ne correspond qu'au préconscient de Freud, ou s'il est encore quelque chose de plus. Les analyses husserliennes de phénomènes tels que la sédimentation, de la même façon que toute la problématique des synthèses passives, interdiront à jamais à Husserl le jugement ironique qu'il avait formulé à l'époque de la Philosophie de l'Arithmétique sur les théories philosophiques de l'inconscient ; qu'elles ne seraient en fait qu'une échappatoire devant l'incapacité à résoudre certains problèmes. Ce que dans la phénoménologie husserlienne pourra intéresser une théorie psychanalytique de l'inconscient c'est le fait qu'elle défend que, par essence, toute conscience est toujours un rapport avec l'inactuel.

\section{BIBLIOGRAPHIE}

BERNET, Rudolf / KERN, Iso / MARBACH, Eduard, Edmund Husserl. Darstellung seines Denkens, Hamburg, Felix Meiner, 1989.

DIEMER, Alwin, Husserl. Versuch einer systematischen Darstellung seiner Phänomenologie, Meisenheim am Glan, Verlag Anton Heim, 1956.

DRÜE, Hermann, Edmund Husserls System der phänomenologischen Psychologie, Berlin, Walter de Gruyter, 1963.

FREUD, Sigmund, Vorlesungen zur Einführung in die Psychoanalyse, in Gesammelte Schriften, Studien Ausgabe, Frankfurt am Main, Suhrkamp, 1982, Bd. I, pp. 37-445.

, Neue Folge der Vorlesungen zur Einführung in die Psychoanalyse, in Gesammelte Schriften, Studien Ausgabe, Band I, pp. 449-608.

, «Triebe und Triebschicksale», in Gesammelte Schriften, Studien Ausgabe, Band III, pp.

81-102.

, Das Ich und das Es, in Gesammelte Werke, Studien Ausgabe, Band III, pp. 282-325.

, «Die Verneinung», in Gesamelte Werke, Studien Ausgabe, Band. III, pp. 373-377.

HOLENSTEIN, Elmar, Phänomenologie der Assoziation, Den Haag, Martinus Nijhoff, 1972.

HUSSERL, Cartesianische Meditationen, Husserliana, Band I, Den Haag, Martinus Nijhoff, 1950. Edmund, Ideen zu einer reiner Phänomenologie und phänomenologischen Philosophie, Zweiter Teil, Husserliana, Band IV, Den Haag, Martinus Nijhoff, 1952.

Analysen zur passiven Synthesis, Husserliana, Band XI, Den Haag, Martinus Nijhoff, 1966.

, Zur Phänomenologie der Intersubjektivität, Husserliana, Band XIII, Den Haag, Martinus

Nijhoff, 1973. 
, Ding und Raum, Husserliana, Band XVI, Den Haag, Martinus Nijhoff, 1973. LANDGREBE, Ludwig, «Prinzipien der Lehre von Empfinden», in Der Weg der Phänomenologie, Güttersloh, Gerd Mohn, 1963, pp. 111-123.

, «Die Phänomenologie der Leiblichkeit und das Problem der Materie», in Phänomenologie und Geschichte, Gütersloh, Gerd Mohn, 1968, pp. 135-147.

PINTOS-PEÑARANDA, Maria da Luz, «Aron Gurwitsch: fenomenologia de la percepción y encuentro con Kurt Goldstein», Phainomenon, 14 (2007) 255-277.

SCHUTZ, Alfred, «Husserl's Ideas, Volume II», in Collected Papers, The Hague, Martinus Nijhoff, 1975, III, pp. 15-39.

SOTO, Francisco Conde, «Temporalidad y intencionalidade passiva en los manuscritos C», Phainomenon, 13 (2006) 37-78.

WELSH, Talia, «The retention and the repressed: does Freud's concept of the Unconscious threaten husserlian phenomenology?», in Human Studies, 25 (2002) 165-183.

\section{NOTES}

1. Cf. LANDGREBE, Ludwig, «Prinzipien der Lehre von Empfinden», in Der Weg der Phänomenologie, Güttersloh, Gerd Mohn, 1963, pp. 111-123, p. 112.

2. Cf. SCHUTZ, Alfred, «Husserl's Ideas, Volume II», in Collected Papers, The Hague, Martinus Hijhoff, 1975, III, pp. 15-39, p. 24.

3. HUSSERL, Edmund, Ding und Raum, § 20, Hua XVI, pp. 64-65.

4. HUSSERL, Edmund, Ideen II, § 41 b), Hua IV, p. 159: ein unvollkommen konstituiertes Ding.

5. HUSSERL, Edmund, Phänomenologische Psychologie, Hua IX, p. 145.

6. Je traduis de cette façon, avec un peu de liberté, l'expression allemande Brüche der Einstimmigkeit.

7. Sur la doctrine husserlienne de l'apprésentation on lira le $\S 50$ des Cartesianische Meditationen, Hua I, pp. 138-141.

8. HUSSERL, Edmund, Ideen II, § 33, Hua IV, 136-137.

9. FREUD, Sigmund, Hemmung, Symptom und Angst, in Gesammelte Schriften, Studien Ausgabe, Bd. VI, Frankfurt am Main, Suhrkamp, 1982, pp. 233-308, p. 274.

10. LANDGREBE, Ludwig, «Prinzipien der Lehre von Empfinden», p. 118.

11. FREUD, Sigmund, Neue Folge der Vorlesungen zur Einführung in die Psychoanalyse, in Gesammelte Schriften, Studien Ausgabe, Bd. I, pp. 449-608, p. 497.

12. DIEMER, Alwin, Husserl. Versuch einer systematischen Darstellung seiner Phänomenologie, Meisenheim am Glan, Verlag Anton Heim, 1956, p. 233.

13. HUSSERL, Edmund, Ideen II, Hua IV, p. 68.

14. Idem, Ibidem, p. 149.

15. LANDGREBE, Ludwig, «Prinzipien der Lehre von Empfinden», p. 115.

16. Cf. HOLENSTEIN, Elmar, Phänomenologie der Assoziation, Den Haag, Martinus Nijhoff, 1972, p. 84. Sur le rapport entre association et synthèse, cf. Husserl, Analysen zur passiven Syntesis (dorénavant : APS), Hua XI, Beilage XVII, pp. 405-411.

17. HUSSERL, Edmund, APS, Hua XI, § 28, pp. 128-133, Beilage XVIII, pp. 405-411. Francisco Conde Soto, in «Temporalidad y intencionalidade passiva en los manuscritos C», Phainomenon, 13 (2006) $37-78$, p. 61, nie qu'on puisse parler déjà de synthèse, comme je l'ai fait plus haut, à ce niveau très élémentaire. Cependant la différence n'est peut-être que d'ordre terminologique. D'ailleurs l'auteur parle aussi de rapports de recouvrement. Notons que la même structure temporelle, 
composée par des phénomènes de rétention et de protension, qui est présente dans les synthèses de recouvrement, se trouve également dans les phénomènes que je viens d'analyser.

18. Je traduis par «réverbération» le mot allemand Resonanz; cf. APS, Hua XI, p. 406.

19. Cf. FREUD, Sigmund, Vorlesungen zur Einführung in die Psychoanalyse, in Gesammelte Schriften, Studien Ausgabe, Bd. I, pp. 37-445, p. 402. Cette comparaison a lieu dans le contexte d'une analyse du narcissisme, ce qui est sans importance pour notre propos actuel.

20. FREUD, Sigmund, «Die Verneinung», in Gesamelte Werke, Studien Ausgabe, Band. III, pp. 373-377, p. 376.

21. HUSSERL, Edmund, Ideen III, Hua V, p. 14.

22. Idem, Grundprobleme der Pnhänomenologie (Vorlesung 1910-1911), in Zur Phänomenologie der Intersubjektivität, Hua XIII, p. 119.

23. Idem, Ibidem, p. 143.

24. Idem, Zur Phänomenologie der Intersubjektivität, p. 21.

25. Idem, APS, Hua XI, pp. 131-132.

26. Idem, APS, pp. 152-153.

27. C'est très peu probable que Husserl se réfère aux théories de G. T. Fechner ou de Ed. von Hartmann. Par contre, il avait fait la connaissance des théories de Carl Jung, dont il avait un livre dans sa bibliothèque.

28. FREUD, Sigmund, Neue Folge der Vorlesungen zur Einführung in die Psychoanalyse, in Gesammelte Schriften, Studien Ausgabe, Band I, p. 496.

29. HUSSERL, Edmund, APS, § 37, Hua XI, p. 177.

30. FREUD, Sigmund, Das Ich und das Es, in Gesammelte Schriften, Studien Ausgabe, Bd. III, pp. $282-325$, p. 289 , n. 1.

\section{RÉSUMÉS}

Este ensaio tem como objectivo apresentar, num primeiro momento, os resultados da análise intencional do processo de constituição dos objectos físicos, do corpo somático e da psique, tal como ela foi levada a cabo por Edmund Husserl. Na base desses resultados, será possível elaborar uma teoria da afecção que permitirá, num segundo momento, algumas aproximações com a Psicanálise de Freud e a teoria freudiana das pulsões. A especificidade do corpo somático relativamente a qualquer objecto físico - apesar de algumas semelhanças no seu modo de doação - revela-se, acima de tudo, na experiência do corpo próprio e nas modalidades de vivência das afecções. Em particular, o enfraquecimento ou obscurecimento destas últimas colocam a questão da existência de um «inconsciente fenomenológico», cujas relações com o inconsciente de Freud, num terceiro e último momento do ensaio, se tentarão pôr em evidência.

This paper addresses, in the first place, the outcomes of the intentional analysis of the constitution of physical objects, the somatic body and the mind, as it was carried out by Edmund Husserl. On the basis of these outcomes, it will be possible to develop a phenomenological theory of affections that will allow, in the second place, some approaches to Freud's Psychoanalysis and the Freudian theory of instincts. The specificity of the somatic body regarding any physical object - despite some similarities in their modes of giveness - is revealed, above all, in one's experience of the somatic body and in one's lived experience of affections. In particular, what Husserl calls the weakening or obscuring process of affections poses the question of the existence 
of a "phenomenological unconscious», whose relations with Freud's unconscious, in a third and final moment of the paper, we will attempt to highlight.

\section{INDEX}

Keywords : living body, lived experience, drive, unconscious

Palavras-chave : corpo somático, experiência vivida, pulsão, inconsciente

\section{AUTEUR}

\section{CARLOS MORUJÃO}

Universidade Católica Portuguesa - Lisboa, Portugal. carlosmorujao@fch.lisboa.ucp.pt

Professor Catedrático na Faculdade de Ciências Humanas da Universidade Católica Portuguesa e Coordenador do Centro de Estudos de Filosofia. A sua área principal de investigação é a Fenomenologia de Husserl, sobre a qual tem ensinado e escrito nos últimos anos. É também o tradutor para português (em colaboração com Pedro M. S. Alves) das Investigações Lógicas de Husserl. Coordena o Seminário Permanente de Fenomenologia e Psicanálise e é investigador do projecto "Corpo e afectividade na fenomenologia material de Michel Henry".

Full Professor at the Portuguese Catholic University, and Director of the Research Unit "Center of Philosophical Studies". His main area of interest is Husserl's phenomenology, about which he has lectured and written extensively in the last years. He is also the translator into Portuguese (in collaboration with Pedro M. S. Alves) of Husserl's Logical Investigations. He coordinates na Ongoing Seminar about the relations between Phenomenology and Psychoanalysis, and a member of the research project «Body and affections in Michel Henry's material phenomenology». 